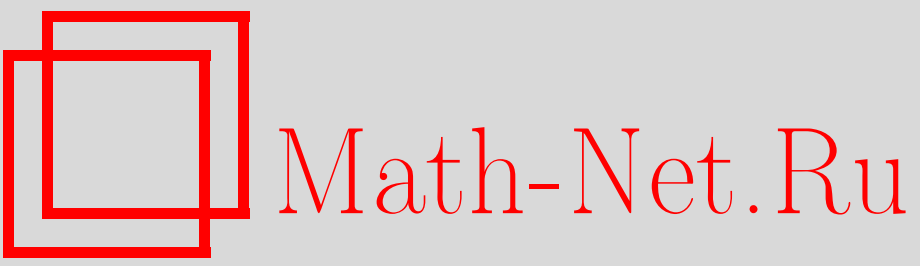

А. М. Левин, М. А. Ольшанецкий, Неавтономные гамильтоновы системы, связанные с высшими интегралами Хитчина, ТМФ, 2000, том 123, номер 2, 237-263

DOI: https://doi.org/10.4213/tmf600

Использование Общероссийского математического портала Math-Net.Ru подразумевает, что вы прочитали и согласны с пользовательским соглашением

http://www . mathnet.ru/rus/agreement

Параметры загрузки:

IP : 54.198 .187 .58

26 апреля 2023 г., 17:39:13 
ТЕОРЕТИЧЕСКАЯ

И МАТЕМАТИЧЕСКАЯ

ФИЗИКА

Том 123, № 2

май, 2000

(C) 2000 г.

А.М. Левин ${ }^{*}$, М. А. Ольшанецкий ${ }^{\dagger}$

\title{
НЕАВТОНОМНЫЕ ГАМИЛЬТОНОВЫ СИСТЕМЫ, СВЯЗАННЫЕ С ВЫСШИМИ ИНТЕГРАЛАМИ ХИТЧИНА
}

\begin{abstract}
Описываются неавтономные гамильтоновы системы, возникающие из интегрируемых систем Хитчина. Интегралы движения Хитчина зависят от $\mathcal{W}$-структур базисной кривой. Параметры $\mathcal{W}$-структур играют роль времен. В частности, квадратичные интегралы зависят от комплексной структуры $\left(\mathcal{W}_{2}\right.$-структуры) базисной кривой, а времена являются координатами на пространстве Тейхмюллера. Соответствующие потоки являются сохраняющими монодромию уравнениями, такими как уравнения Шлезингера, уравнения Пенлеве VI и их обобщения. Уравнения, отвечающие высшим интегралам, являются условиями сохранения монодромии при изменении $\mathcal{W}_{k}$-структур $(k>2)$. Они выводятся посредством симплектической редукции из калибровочной теории поля на базисной кривой, взаимодействующей с $\mathcal{W}_{k}$-гравитацией. Как следствие из предложенной теории получаются классические тождества Уорда.
\end{abstract}

Памяти Михаила Савельева

\section{1. ВВЕДЕНИЕ}

Соответствующие $\mathcal{W}$-алгебрам бесконечномерные симметрии играют определенную роль в двумерной теории поля (см. обзор в работе [1]). В настоящей работе мы исследуем классические гамильтоновы системы, включенные в калибровочную теорию на римановой кривой, взаимодействуюшей с $\mathcal{W}$-гравитацией. В то время как $\mathcal{W}_{2}$-гравитация имеет естественную геометрическую основу, нет удовлетворительного геометрического понимания происхождения $\mathcal{W}_{k}$-теории при $k>2$. Сушествует точка зрения на $\mathcal{W}_{k}$-гравитацию как на геометрию некоторых двумерных поверхностей, вложенных в $k$-мерное афффинное пространство. Одна из работ М. Савельева [2] представляет собой важный шаг в этом направлении. В настояшей работе мы анализируем связь между $\mathcal{W}$-геометрией и интегрируемыми системами. На исследование последней проблемы М. Савельев оказал сушественное влияние.

Рассмотрим подкласс классических полностью интегрируемых систем с фазовыми

*Max-Planck-Institut für Mathematik, Вonn; Институт океанологии, Москва, Россия. E-mail: andrl@landau.ac.ru

${ }^{\dagger}$ Max-Planck-Institut für Mathematik, Bonn; Институт теоретической и экспериментальной физики, Москва, Россия. E-mail: olshanet@heron.itep.ru 
потоками, имеющими лаксов вид,

$$
\partial_{s} L=\left[L, M_{s}\right], \quad \partial_{s}=\frac{\partial}{\partial t_{s}} .
$$

Будем считать, что:

1) оператор $L$ принимает значения в комплексной алгебре Ли. Мы ограничимся случаем $s l(N, \mathbb{C})$;

2) $L=L(z)$, где $z$-спектральный параметр, лежаший на римановой кривой $\Sigma_{g, n}$ рода $g$ с $n$ отмеченными точками; $L$ может иметь полюсы первого порядка в отмеченных точках. Таким образом, мы исключаем из дальнейшего рассмотрения явление Сток$\mathrm{ca}^{1)}$.

Интегрируемые системы на кривых без отмеченных точек рассматривались Хитчиным [4]. В этом случае нетривиальные уравнения возникают только на кривых старшего рода $(g>1)$. До недавнего времени не было явных примеров интегрируемых систем такого типа ${ }^{2}$. Обобщение подхода Хитчина на матрицы с полюсами первого порядка в отмеченных точках [7] позволило включить в данный подход некоторые хорошо известные полностью интегрируемые модели, такие как модели Тоды и системы Калоджеро-Мозера. Квантовым аналогом этих систем являются уравнения Книжника-Замолодчикова-Бернара для конформных блоков в теории Весса-Зумино-Виттена (ВЗВ) на критическом уровне, в то время как собственно системы Хитчина соответствуют уравнениям для статсумм этих теорий.

В системах типа систем Хитчина выражения $\operatorname{tr}\left(L^{k}\right)=\left\langle L^{k}\right\rangle$ преврашаются в интегралы движения после интегрирования по $\Sigma_{g, n}$. До интегрирования необходимо учесть, что операторы $L$ являются $(1,0)$-формами на $\Sigma_{g, n}$ в некоторой фиксированной комплексной структуре. Для интегрирования $\left\langle L^{k}\right\rangle$ необходимо умножить его на $(-k+1,1)$-дифференшиал $\rho_{k, s}=\rho_{k, s}(z, \bar{z}) \partial_{z}^{k-1} \otimes d \bar{z}$. Индекс $s$ возникает следуюшим образом. Оператор $\rho_{k, s}(z, \bar{z}) \partial_{z}^{k-1}$ определен в локальных координатах точки $(z, \bar{z})$. Те из них, которые представимы как $\bar{\partial}$-производные, не вносят вклада в гамильтонианы. Таким образом, можно выбрать $\rho_{k, s}$ лежашими в $H^{1}\left(\Sigma_{g}, \Gamma^{k-1}\right)$, где $\Gamma$ - векторные поля на $\Sigma_{g, n}$, обрашаюшиеся в нуль в отмеченных точках. Тогда индекс $s$ нумерует базис в $H^{1}\left(\Sigma_{g}, \Gamma^{k-1}\right)$. Представим дифференциалы в виде

$$
\rho_{k, s}=t_{k, s} \rho_{s, k}^{0},
$$

где $\left\{\rho_{s, k}^{0}\right\}-$ фиксированный базис в $H^{1}\left(\Sigma_{g, n}, \Gamma^{k-1}\right)$. Интегралы движения принимают вид

$$
H_{k, s}=\frac{1}{k} \int_{\Sigma_{g}}\left\langle L^{k}\right\rangle \rho_{k, s}^{0} \quad\left(k=1, \ldots, N, \quad s=1, \ldots, \operatorname{dim} H^{1}\left(\Sigma_{g}, \Gamma^{k-1}\right)\right) .
$$

Важный класс гамильтоновых уравнений возникает, если $t_{k, s}$ рассматриваются как "времена". В этом случае $H_{k, s}$ становятся гамильтонианами неавтономных систем, времена которых связаны с деформациями интегральных структур базисной кривой $\Sigma_{g, n}$.

\footnotetext{
1) Это ограничение было частично снято в недавней работе [3].

${ }^{2)} \mathrm{Cм}$., однако, работы $[5,6]$ для $g=2, L \in \operatorname{sl}(2, \mathbb{C})$.
} 
Оказывается, что фазовые потоки этих систем описьваются следующей модификацией уравнения Лакса (1.1):

$$
\partial_{a} L-\partial M_{a}+\left[M_{a}, L\right]=0, \quad a=(k, s), \quad \partial=\frac{\partial}{\partial z} .
$$

Рассмотрим сначала квадратичные гамильтонианы $H_{2, s}$. В этом случае $\rho_{2, s}=\mu_{s}$ являются $(-1,1)$-дифференциалами (дифференциалами Бельтрами), это $(0,1)$-формы со значениями в векторных полях на $\Sigma_{g, n}$. Мы рассматриваем здесь алгебру Ли векторных полей и группу локальных диффеоморфизмов $\Sigma_{g, n}$. Грубо говоря, пространство $H^{1}\left(\Sigma_{g, n}, \Gamma\right)$ можно определить как пространство гладких $(-1,1)$-дифференциалов на $\Sigma_{g, n}$, рассматриваемых по модулю глобальных диффеоморфизмов. Элементы из $H^{1}\left(\Sigma_{g, n}, \Gamma\right)$ играют роль параметров деформации комплексной структуры на $\Sigma_{g, n}$. В случае $g=0$ комплексная структура определяется положениями отмеченных точек $t_{s}=x_{s}-x_{s}^{0}$, и уравнения (1.4) приводят к системе Шлезингера. Другие интересные примеры, включаюшие, в частности, семейство уравнений Пенлеве VI, возникают, если базисная кривая является эллиптической кривой с отмеченными точками [8].

При $k>2$ дифференциалы $\rho_{k, s}$ не порождают алгебру Ли. Из-за этого они не имеют естественного геометрического описания. Вместе с дуальными объектами (операми [9]) они определяют так называемую $\mathcal{W}_{k}$-геометрию базисной кривой $\Sigma_{g, n}[10,11]$. $\mathcal{W}_{k}$-геометрия является обобшением $\mathcal{W}_{2}$-геометрии, которая совпадает с пространством проективных структур на $\Sigma_{g, n}$.

Инвариантным объектом, связанным с лаксовой формой (1.1) интегрируемых систем, является спектральная кривая $\mathcal{C}: \operatorname{det}(\lambda+L(z))=0$. Эта кривая представляет собой $N$-накрытие базисной кривой $\Sigma_{g, n}$. Многообразие Прима $\operatorname{Prym}\left(\mathcal{C} / \Sigma_{g}\right)$ является лиувиллевым тором полностью интегрируемой системы (1.1) [4]. Имеются различные параметризации кривой $\mathcal{C}$. Две стандартные параметризации представляют собой множества гамильтонианов $H_{k, s}(1.3)$ и переменных действия. С помощью дифференциалов $\rho_{k, s}(1.2)$ получается другая параметризация $\mathcal{C}$, связанная с $\mathcal{W}_{N}$-структурой базисной кривой. Для малых времен уравнение (1.4) описывает эволюцию $\mathcal{C}$ вблизи фиксированной кривой $\mathcal{C}_{0}$.

Основная цель настояшей работы заключается в исследовании динамических систем (1.4), связанных с $\mathcal{W}_{k}$-геометрией. $\mathrm{B}$ таком исследовании имеется два важных аспекта. Во-первых, квантовым аналогом этих систем являются уравнения КнижникаЗамолодчикова-Бернара высшего порядка вне критического уровня. Это означает, что конформные блоки в теории ВЗВ удовлетворяют некоторому аналогу нестационарных уравнений Шредингера с операторами Казимира высшего порядка и временами $t_{k, s}$, $k>2$. Нам неизвестны какие-либо явные примеры уравнений такого типа. Во-вторых, исследование высших $\mathcal{W}$-геометрий интересно само по себе. Такая задача не простая, поскольку эти геометрии не являются геометриями Клейна - как мы уже отмечали, нет очевидных групп симметрий, связанных с ними. Связь $\mathcal{W}$-геометрий с интегрируемыми системами открывает новый путь для их исследований, а именно можно использовать подходы, разработанные для интегрируемых систем, например квантовый метод Уизема [12], который применим к анализу возмушений интегралов движения (1.3). 
Наше построение основано на подходе, развитом в работе [10]. $\mathcal{W}_{k}$-структуры описываются там как результат гамильтоновой редукции относительно максимальной параболической подгруппы в $S L(k, \mathbb{C})$. Мы обобшаем этот подход в двух направлениях:

a) рассматриваем римановы кривые с отмеченными точками и с некоторыми данными в этих точках;

б) в дополнение к $\mathcal{W}$-полям включаем в рассмотрение $S L(N, \mathbb{C})$-калибровочные поля.

В результате получаются классические тождества Уорда для $\mathcal{W}$-гравитации, взаимодействующей с калибровочными теориями на римановых кривых с отмеченными точками. Чтобы получить сохраняюшие монодромию потоки, мы исключаем половину $\mathcal{W}$-полей, оставляя только дифференциалы $\rho_{k, s}$, которые играют роль “времен”. Такая процедура также основана на гамильтоновой редукции относительно симметрий, порождаемых связями типа связей Шугавары. На этом этапе мы приходим к свободным системам с тривиальными потоками. В итоге гамильтонова редукция, основанная на калибровочной $S L(N, \mathbb{C})$-симметрии, приводит к нетривиальным неавтономным системам.

Работа построена следуюшим образом. В разделах 2 и 3 дается вывод сохраняюших монодромию уравнений с использованием проективных структур на базисных кривых. Далее (раздел 4) аналогичный подход применяется, с бо́льшими подробностями, к случаю $\mathcal{W}_{3}$-структур. В разделе 5 мы кратко обсуждаем общий случай.

\section{2. ПРОЕКТИВНЫЕ СТРУКТУРЫ НА РИМАНОВЫХ КРИВЫХ И СИМПЛЕКТИЧЕСКАЯ ГЕОМЕТРИЯ}

2.1. Проективные структуры. Фиксируем комплексную структуру на $\Sigma_{g, n}$, выбрав пару локальных координат $(z, \bar{z})$ и соответствуюшие операторы $(\partial, \bar{\partial})$. Деформированная комплексная структура может быть получена из решений уравнения Бельтрами

$$
(\bar{\partial}+\mu \partial) F=0 .
$$

Локально $F(z, \bar{z})$ представляет собой диффеоморфизм

$$
w=F(z, \bar{z}), \quad \bar{w}=\bar{F}(z, \bar{z}),
$$

а дифференциал Бельтрами

$$
\mu(z, \bar{z})=-\frac{\bar{\partial} \bar{F}}{\partial F}
$$

определяет оператор новой комплексной структуры $\partial_{\bar{w}}:=\bar{\partial}+\mu \partial$. Два дифференциала Бельтрами дают эквивалентные комплексные структуры, если они связаны глобальным голоморфным диффеоморфизмом кривой $\Sigma_{g, n}$

$$
w=z-\epsilon(z, \bar{z}), \quad \bar{w}=\bar{z} .
$$

Мы полагаем, что алгебра Ли $\mathcal{V}_{g, n}$ соответствующих векторных полей на $\Sigma_{g, n}$ задается их поведением вблизи отмеченных точек:

$$
\mathcal{V}_{g, n}=\left\{\epsilon(z, \bar{z}) \partial \mid \epsilon(z, \bar{z})=O\left(z-x_{a}\right)\right\} .
$$


При голоморфных диффеоморфизмах $\mu$ преобразуется как $(-1,1)$-дифференциал. Векторные поля действуют на $\mu$ как

$$
j_{\epsilon} \mu=\epsilon \partial \mu-\mu \partial \epsilon-\bar{\partial} \epsilon
$$

Определим зависимость $\mu$ от положения отмеченных точек следуюшим образом. Пусть $\mathcal{U}_{a}^{\prime}$ - окрестности отмеченных точек $x_{a}(a=1, \ldots, n)$, так что $\mathcal{U}_{a}^{\prime} \cap \mathcal{U}_{b}^{\prime}=\varnothing$ при $a \neq b$. Определим гладкую функцию

$$
\chi_{a}(z, \bar{z})= \begin{cases}1, & z \in \mathcal{U}_{a}, \quad \mathcal{U}_{a}^{\prime} \supset \mathcal{U}_{a} \\ 0, & z \in \Sigma_{g} \backslash \mathcal{U}_{a}^{\prime}\end{cases}
$$

Введем времена, связанные с положениями отмеченных точек $t_{2, a}=x_{a}-x_{a}^{0}$. Тогда $\mu$ можно представить в виде

$$
\mu=\sum_{a=1}^{n} t_{2, a} \mu_{a}^{0}, \quad \mu_{a}^{0}=\bar{\partial} \epsilon_{a}(z, \bar{z}), \quad \epsilon_{a}(z, \bar{z})=\chi_{a}(z, \bar{z}), \quad t_{2, a}=x_{a}-x_{a}^{0} .
$$

Пусть $T$ - проективная связность на $\Sigma_{g, n}$, т.е. $T$ преобразуется при голоморфиых диффеоморфизмах как $(2,0)$-дифференциал с точностью до добавления производной Шварца. Локально это означает, что

$$
j_{\epsilon} T(z, \bar{z})=\epsilon \partial T+2 T \partial \epsilon-\frac{\kappa^{2}}{2} \partial^{3} \epsilon
$$

где $\kappa$-параметр, который в дальнейшем будет играть роль "постоянной Планка" в квантовании Уизема. Примем, что $T$ имеет полюсы в отмеченных точках $x_{a}(a=1, \ldots, n)$ вплоть до второго порядка:

$$
\left.T\right|_{z \rightarrow x_{a}} \sim \frac{T_{-2, a}}{\left(z-x_{a}\right)^{2}}+\frac{T_{-1, a}}{\left(z-x_{a}\right)}+\cdots .
$$

Пусть $\widetilde{\mathcal{W}}_{2}$-пространство пар $(T, \mu)$ на $\Sigma_{g, n}$, причем поведениевблизи отмеченных точек определяется условиями (2.6) и (2.8).

ОпРЕДЕЛЕнИЕ 1 . Пространство $\mathcal{W}_{2}$ проективной структуры на $\Sigma_{g, n}$ является подмножеством в $\widetilde{\mathcal{W}}_{2}$, удовлетворяюшим уравнению

$$
(\bar{\partial}+\mu \partial+2 \partial \mu) T=\frac{1}{2} \partial^{3} \mu,
$$

с фиксированными значениями $\mathbf{T}_{2}=\left(T_{-2,1}, \ldots, T_{-2, n}\right)$ из $(2.8)$.

Пусть $\psi-(-1 / 2,0)$-дифференциал. Тогда уравнение (2.9) представляет собой условие совместности для линейной системы

$$
\begin{gathered}
\left(\bar{\partial}+\mu \partial-\frac{1}{2} \partial \mu\right) \psi=0 \\
\left(\partial^{2}-T\right) \psi=0
\end{gathered}
$$

3 Теоретическая и математическая физика, т. 123, № 2, 2000 г. 
Рассмотрим два линейно независимых решения $\psi_{1}, \psi_{2}$ этой системы. Проективная структура $(T, \mu)$ может быть эквивалентным образом определена отношением $F=$ $\psi_{1} / \psi_{2}$. Действительно, как следует из уравнения (2.10), $F$ удовлетворяет уравнению Бельтрами (2.9). Следовательно, $\mu=\bar{\partial} F / \partial F$. С другой стороны, из уравнения (2.11) следует, что $\psi_{1}=(\partial F)^{1 / 2}$ и $T=\mathcal{S}_{z}(F)$, где $\mathcal{S}_{z}(F)$ - производная Шварца от $F$. Произвольные линейно независимые решения системы $(2.10),(2.11)$ получаются из $\psi_{1}, \psi_{2}$ с помошью $S L(2, \mathbb{C})$-преобразований. Они дают преобразование Мёбиуса отношения $F$, а $T=\mathcal{S}_{z}(F)$ при этом не изменяется. Таким образом, отношение любых двух независимых решений определяет проективную структуру.

2.2. Симплектическая редукция относительно диффеоморфизмов. Пространство $\mathcal{W}_{2}$ аналогично пространству плоских связностей на $\Sigma_{g, n}$ для некоторой калибровочной группь. Это симплектическое многообразие, которое можно получить из афффинного пространства гладких связностей путем симплектической редукции. Условие того, что связности плоские, играет роль сохраняющегося момента при симплектической редукции. Аналогичную процедуру можно применить к пространству $\widetilde{\mathcal{W}}_{2}$, что дает $\mathcal{W}_{2}$. В этом случае калибровочная группа заменяется на группу голоморфных диффеоморфизмов кривой $\Sigma_{g, n}(2.2)$. Пространство $\widetilde{\mathcal{W}}_{2}$ может быть снабжено симплектической структурой

$$
\omega=-\kappa^{-1} \int_{\Sigma_{g}} \delta T \delta \mu
$$

Векторные поля $\mathcal{V}_{g, n}$, определенные в $(2.4)$ и $(2.7)$, действуют как симметрии формы $\omega$. Гамильтониан этого действия

$$
H_{\epsilon}=-\kappa^{-1} \int_{\Sigma_{g}} \epsilon\left[(\bar{\partial}+\mu \partial+2 \partial \mu) T-\frac{\kappa^{2}}{2} \partial^{3} \mu\right]
$$

приводит к отображению моментов

$$
m: \widetilde{W}_{2} \rightarrow \mathcal{V}_{g, n}^{*}, \quad m=(\bar{\partial}+\mu \partial+2 \partial \mu) T-\frac{\kappa^{2}}{2} \partial^{3} \mu,
$$

где $\mathcal{V}_{g, n}^{*}-$ пространство, дуальное к алгебре $\mathcal{V}_{g, n}$ векторных полей, т.е. пространство $(2,1)$-форм на $\Sigma_{g, n}$. Как следует из $(2.3)$, в окрестностях отмеченных точек элементы $y \in \mathcal{V}_{g, n}^{*}$ принимают вид

$$
y \sim b_{1, a} \partial \delta\left(x_{a}\right)+b_{2, a} \partial^{2} \delta\left(x_{a}\right)+\cdots .
$$

Таким образом, члены $T_{-1, a} \delta\left(x_{a}\right)$, появляюшиеся в члене $\bar{\partial} T$ (уравнение $\left.(2.13)\right)$ из полюсов $T$ первого порядка (см. (2.8)), выпадают из отображения моментов $m$, определяемого уравнением (2.13). Положим

$$
m=-\sum_{a=1}^{n} T_{-2, a} \partial \delta\left(x_{a}\right)
$$

Из $(2.8),(2.13)$ и $(2.14)$ следует, что в $(2.14)$ следует взять $b_{1, a}=-T_{-2, a}, b_{k, a}=0$, $k>1$. Таким образом, условие $(2.9)$, которое выделяет $\mathcal{W}_{2}$ в $\widetilde{\mathcal{W}}_{2}$, представляет собой условие сохраняюшегося момента относительно действия диффеоморфизмов. 
2.3. Подход “Дринфельда-Соколова”. В работе [10] была предложена другая процедура, похожая на подход Дринфельда-Соколова. Вкратце она выглядит следующим образом. Рассмотрим аффинное пространство $\mathcal{N}_{2}$ гладких плоских $S L(2, \mathbb{C})$-связностей на $\Sigma_{g, n}$ :

$$
\begin{aligned}
\mathcal{N}_{2} & =\{a d z+\bar{a} d \bar{z}\}, \\
F(a, \bar{a}) & =\bar{\partial} a-\partial \bar{a}+[a, \bar{a}]=0 .
\end{aligned}
$$

Поле $а$ имеет полюсы в отмеченных точках до второго порядка. Пространство $\mathcal{N}_{2}$ имеет стандартную симплектическую форму

$$
\omega^{\prime}=\int_{\Sigma_{g, n}} \operatorname{tr}(\delta a \delta \bar{a}) .
$$

Форма инвариантна при калибровочных преобразованиях

$$
a \rightarrow g^{-1} a g+g^{-1} \partial g, \quad \bar{a} \rightarrow g^{-1} \bar{a} g+g^{-1} \bar{\partial} g, \quad g \in \mathcal{G}=\operatorname{Map}\left(\Sigma_{g, n}, S L(2, \mathbb{C})\right) .
$$

Будем считать, что алгебра Ли $\operatorname{Lie}(\mathcal{G})$ калибровочной группы $\mathcal{G}$ определяется поведением своего матричного элемента $x^{12}$ вблизи отмеченных точек:

$$
\left.x^{12}\right|_{z \rightarrow x_{a}}=O\left(z-x_{a}\right), \quad x^{J K} \in \operatorname{Lie}(\mathcal{G}) .
$$

Условие нулевой кривизны (2.15) является отображением моментов для действия калибровочной группы. Форма $\omega^{\prime}$ вырождена - она обращается в нуль на орбитах калибровочной группы, потому что мы налагаем только условие моментов (2.15) и не фиксируем калибровку. Если же мы сделаем это, то получим конечномерное пространство плоских связностей, однако нам надо сохранить два поля $T$ и $\mu$. Прием, предложенный в работе [10], заключается в фиксировании калибровки относительно борелевской подгруппы $B$ нижнетреугольных матриц. В [10] было показано, что это позволяет редуцировать пространство $\mathcal{N}_{2}$ до пространства проективных связностей $\mathcal{W}_{2}$.

Калибровочная свобода позволяет фиксировать матрицу $a$ общего положения в виде

$$
a=\left(\begin{array}{cc}
0 & 1 \\
T & 0
\end{array}\right)
$$

где $T$ - новое поле, удовлетворяющее условию (2.8). Полюсы $T$ первого порядка не вносят вклада в уравнение моментов (2.15), поскольку они пропадают при калибровочном преобразовании (см. (2.17)). Уравнение моментов (2.15) позволяет выразить все матричные элементы $\bar{a}$ в терминах $T$ и нового поля $\mu$ :

$$
\bar{a}=\left(\begin{array}{cc}
\frac{1}{2} \partial \mu & -\kappa^{-1} \mu \\
-\kappa^{-1} \mu T+\frac{1}{2} \kappa \partial^{2} \mu & -\frac{1}{2} \partial \mu
\end{array}\right) .
$$

Условие нулевой кривизны становится тривиальным для всех матричных элементов, кроме $F_{(2,1)}$. Можно проверить, что оно совпадает с условием проективности $(2.9)$. 
Линейная система для $(-1 / 2,0)$-дифференциалов $\psi$

$$
(\bar{\partial}-\bar{a})\left(\begin{array}{c}
\psi \\
\kappa \partial \psi
\end{array}\right)=0, \quad(\kappa \partial-a)\left(\begin{array}{c}
\psi \\
\kappa \partial \psi
\end{array}\right)=0
$$

является совместной согласно уравнению (2.15). Она является матричной формой уравнений $(2.10)$ и (2.11) для специального вида $a(2.18)$ и $\bar{a}(2.19)$.

Исходная симплектическая структура $\omega^{\prime}(2.16)$ редуцируется к $\omega(2.12)$ на пространстве проективных структур $\mathcal{W}_{2}$.

Хотя при таком подходе диффеоморфизмы явно не возникают, они присутствуют в данной конструкции скрытым образом. Чтобы показать это, вычислим коммутатор двух матрищ $\bar{a}_{1}, \bar{a}_{2}$ :

$$
\left[\bar{a}_{1}\left(\mu_{1}, T_{1}\right), \bar{a}_{2}\left(\mu_{2}, T_{2}\right)\right]_{(1,2)}=\mu_{1} \partial \mu_{2}-\mu_{2} \partial \mu_{1}
$$

Таким образом, коммутатор матриц $\bar{a}$ воспроизводит коммутатор векторных полей.

\section{3. ИЗОМОНОДРОМНЫЕ ДЕФОРМАЦИИ И ПРОЕКТИВНЫЕ СТРУКТУРЫ}

3.1. Определение $\mathcal{W}_{2}^{N}$-структур. Рассмотрим некоторую проективную структуру на $\Sigma_{g, n}$, определенную линейной системой $(2.10),(2.11)$. Обобщим ее следующим образом. Рассмотрим векторное $S L(N, \mathbb{C})$-расслоение $V$ над $\Sigma_{g, n}$. Пусть $(A, \bar{A})$ - связности в $V$, соответствуюшие комплексной структуре, которую мы выбрали. Будем считать, что:

а) связность $\bar{A}$ является гладкой;

б) связность $A$ имеет полюсы первого порядка в отмеченных точках:

$$
A \sim \frac{A_{-1, a}}{z-x_{a}}+A_{0, a}+\cdots, \quad a=1, \ldots, n .
$$

Кроме этих данных, рассмотрим множество коприсоединенных орбит группы $S L(N, \mathbb{C})$ в отмеченных точках

$$
\left(\mathcal{O}_{1}, \ldots, \mathcal{O}_{n}\right), \quad \mathcal{O}_{a}=\left\{p_{a}=g_{a} p_{a}^{0} g_{a}^{-1} \mid g_{a} \in S L(N, \mathbb{C})\right\},
$$

где $p_{a}^{0}$ определяет орбиту $\mathcal{O}_{a}$. Для приведения этих данных в соответствие с проективными структурами вместо оператора (2.11) мы будем в дальнейшем рассматривать матричный оператор $(\kappa \partial+A)^{2}-T$. Определим матрицы

$$
\begin{aligned}
\widetilde{T} & =T-A^{2}-\kappa \partial A \\
f_{1} & =-\bar{A}+\frac{1}{2} \partial \mu \mathbf{1}_{N}-\frac{1}{\kappa} \mu A .
\end{aligned}
$$

В дальнейшем мы будем опускать в обозначениях умножение на скалярную матрицу $\mathbf{1}_{N}$.

Определим пространство $\widetilde{\mathcal{W}}_{2}^{N}$ полей $(T, \mu),(A, \bar{A}), \mathbf{p}=\left(p_{1}, \ldots, p_{l}\right)$ на $\Sigma_{g, n}$, где поведение $\mu, T$ и $A$ в окрестности отмеченных точек удовлетворяет соответственно условиям $(2.6),(2.8)$ и $(3.1)$. 
ОПредЕлЕниЕ $2 . \mathcal{W}_{2}^{N}$-структура на $\Sigma_{g}$ является подмножеством в $\widetilde{\mathcal{W}}_{2}^{N}$, удовлетворяюшим уравнениям

$$
\begin{gathered}
(\bar{\partial}+\mu \partial+2 \partial \mu) \widetilde{T}-\frac{1}{2} \kappa^{2} \partial^{3} \mu=\left[\widetilde{T}, \bar{A}+\frac{1}{\kappa} \mu A\right]+2 \kappa A \partial f_{1}-\kappa^{2} \partial^{2}\left(\bar{A}+\frac{1}{\kappa} \mu A\right), \\
\bar{\partial} A-\kappa \partial \bar{A}+[A, \bar{A}]=0 \\
A_{-1, a}=-p_{a} \\
T_{-2, a}=\frac{\left\langle\left(p_{a}^{0}\right)^{2}\right\rangle}{N}, \quad a=1, \ldots, n .
\end{gathered}
$$

В первом соотношении правая часть представляет собой вклад калибровочных полей в классическое тождество Уорда для гравитационных полей $(T, \mu)$. Второе равенство представляет собой стандартное условие нулевой кривизны для калибровочных полей. Последнее уравнение выражает наиболее сингулярные члены $T_{-2, a}$ проективной связности $T$ в терминах вторых операторов Казимира, параметризуюших орбиты $\mathcal{O}_{a}$.

Имеется непосредственное обобшение линейной системы (2.10), (2.11), определяюшее проективную структуру. Пусть $\Psi$ - элемент пространства сечений расслоения $\Omega^{(-1 / 2,0)}\left(\Sigma_{g, n}\right.$, Aut $\left.V\right)$.

ПРЕДЛОЖЕНИЕ 1. Уравнения (3.5) и (3.6) являются условиями совместности для линейной системь

$$
\begin{gathered}
\left(\bar{\partial}+\mu \partial-\frac{1}{2} \partial \mu+\bar{A}+\frac{1}{\kappa} \mu A\right) \Psi=0, \\
{\left[(\kappa \partial+A)^{2}-T\right] \Psi=0 .}
\end{gathered}
$$

Пространство $\mathcal{W}_{2}^{N}$ можно снабдить симплектической формой

$$
\omega=\int_{\Sigma_{g, n}}\left[-\frac{N}{\kappa} \delta T \delta \mu+2\langle\delta A, \delta \bar{A}\rangle\right]+4 \pi i \sum_{a=1}^{n} \delta\left\langle p_{a}, g_{a}^{-1} \delta g_{a}\right\rangle .
$$

Последняя сумма в (3.11) представляет собой вклад симплектических форм на коприсоединенных орбитах. Ниже мы покажем, что такая форма является естественной и получается с помощью процедуры гамильтоновой редукции.

3.2. Симплектическая конструкция. Рассмотрим векторное расслоение $V_{2 N}=$ $V_{2} \otimes V_{N}$ над $\Sigma_{g}$ со структурной группой $G=S(G L(2, \mathbb{C}) \otimes G L(N, \mathbb{C})) \sim S L\left(2 N^{2}, \mathbb{C}\right)$. В недеформированной комплексной структуре на $\Sigma_{g, n}$ операторы связности имеют вид $\kappa \partial-\mathcal{A}, \bar{\partial}-\overline{\mathcal{A}}$, где $\mathcal{A}$ и $\overline{\mathcal{A}}$ принимают значения в $\operatorname{Lie}(S(G L(2, \mathbb{C}) \otimes G L(N, \mathbb{C})))$. Первые компоненты $\mathcal{A}$ и $\overline{\mathcal{A}}$ действуют на сечения 1-струй расслоения $\Omega^{(-1 / 2,0)}\left(\Sigma_{g, n}\right)$, а вторыена сечения расслоения $\Omega^{(-1 / 2,0)}\left(\Sigma_{g, n}\right.$, Aut $\left.V\right)$.

Определим $\mathcal{K}_{2}$ как пространство связностей $(\mathcal{A}, \overline{\mathcal{A}})$ и коприсоединенными орбитами в отмеченных точках:

$$
(\mathcal{A}, \overline{\mathcal{A}}),\left(\mathbf{1}_{2} \otimes \mathbf{p}\right), \quad \mathbf{p}=\left(p_{1}, \ldots, p_{n}\right),
$$

и наложим следуюшие дополнительные ограничения: 
а) компоненты $\mathcal{A}$ являются калибровочно-эквивалентными специальной форме

$$
\mathcal{A} \sim D S \otimes \mathbf{1}_{N}+\mathbf{1}_{2} \otimes(-A)
$$

где

$$
D S=\left(\begin{array}{cc}
0 & 1 \\
T & 0
\end{array}\right)
$$

б) вблизи отмеченных точек $T$ имеет вид (2.8);

в) вблизи отмеченных точек $A$ имеет вид (3.1).

Предложение 1 вытекает из следующего утверждения.

ПРЕДЛОЖЕНИЕ 2. $\mathcal{W}_{2}^{N}$ представляет собой подмножсество в $\mathcal{K}_{2}$, удовлетворяющее условию нулевой кривизнь

$$
[\kappa \partial-\mathcal{A}, \bar{\partial}-\overline{\mathcal{A}}]=0
$$

ДокАЗАТЕЛЬСтво. Пространство $\mathcal{K}_{2}$ имеет стандартную симплектическую форму

$$
\omega^{\prime}=\int_{\Sigma_{g, n}}\langle\langle\delta \mathcal{A}, \delta \overline{\mathcal{A}}\rangle\rangle+4 \pi i \sum_{a=1}^{n} \delta\left\langle p_{a}, g_{a}^{-1} \delta g_{a}\right\rangle
$$

где $\langle\langle\cdot, \cdot\rangle\rangle$ - след в тензорном произведении, а $\langle\cdot, \cdot\rangle$ - след в пространстве $V_{N}$.

Введем группу калибровочных преобразований, состоящую из гладких отображений,

$$
\mathcal{G}=\left\{\operatorname{Map}\left(\Sigma_{g, n}, S L\left(2 N^{2}, \mathbb{C}\right)\right)\right\}
$$

с дополнительными ограничениями на отображения вблизи отмеченных точек. Мы сформулируем их для алгебры Ли $\operatorname{Lie}(\mathcal{G})$. Пусть $x_{\alpha \beta}^{I J}$ - матричный элемент в $\operatorname{Lie}(\mathcal{G})$, верхние индексы $I, J$ соответствуют пространству $V_{2}$, а нижние индексы $\alpha, \beta$-пространству $V_{N}$. Примем, что в окрестности отмеченной точки

$$
\left.\operatorname{tr}\right|_{V_{N}} x^{12} \sim O\left(z-x_{a}\right), \quad x_{\alpha \beta}^{12} \sim O\left(z-x_{a}\right)^{2},
$$

а остальные матричные элементы в отмеченных точках непрерывны. Можно проверить, что эти матрицы порождают подалгебру Ли $\operatorname{Lie}(\mathcal{G})$ в алгебре Ли гладких отображений.

Форма $\omega^{\prime}(3.14)$ инвариантна при калибровочных преобразованиях

$$
\begin{gathered}
\overline{\mathcal{A}} \rightarrow f^{-1} \bar{\partial} f-f^{-1} \overline{\mathcal{A}} f, \quad f \in \mathcal{G}, \\
\mathcal{A} \rightarrow f^{-1} \kappa \partial f-f^{-1} \mathcal{A} f, \\
g_{a} \rightarrow g_{a} f_{a}, \quad p_{a} \rightarrow f_{a}^{-1} p_{a} f_{a}, \quad f_{a}=f\left(x_{a}, \bar{x}_{a}\right) .
\end{gathered}
$$

Отождествим дуальное пространство $\operatorname{Lie}^{*}(\mathcal{G})$ с матрицами, существующими на $\Sigma_{g, n}$, при помощи билинейной формы

$$
\int_{\Sigma_{g, n}}\langle\langle x, y\rangle\rangle, \quad x \in \operatorname{Lie}(\mathcal{G}), \quad y \in \operatorname{Lie}^{*}(\mathcal{G}) .
$$


Согласно $(3.15)$ матричные элементы $y \in \operatorname{Lie}^{*}(\mathcal{G})$ имеют в отмеченных точках вид

$$
\begin{aligned}
\left.\operatorname{tr}\right|_{V_{N}} y^{21} & \sim \sum_{k \geqslant 1} b_{k} \partial^{k} \delta\left(x_{a}\right), \quad y_{\alpha \beta}^{21} \sim \sum_{k \geqslant 2} b_{k, \alpha, \beta} \partial^{k} \delta\left(x_{a}\right), \\
y_{\alpha \beta}^{J K} & \sim \sum_{k \geqslant 0} b_{k, \alpha, \beta} \partial^{k} \delta\left(x_{a}\right), \quad J \neq 2, \quad K \neq 1 .
\end{aligned}
$$

Условие (3.12) аналогично частичному фиксированию калибровки. Выберем $\mathcal{A}$ в виде

$$
\mathcal{A}=\left(\begin{array}{cc}
0 & E \\
\widetilde{T} & -2 A
\end{array}\right)
$$

где $\widetilde{T}$ задано в (3.3). Такой вид $\mathcal{A}$ можно получить калибровочным преобразованием (3.12) с помошью

$$
f=\left(\begin{array}{cc}
\mathbf{1}_{N} & 0 \\
A & \mathbf{1}_{N}
\end{array}\right) .
$$

Как обычно, тензор кривизны $F(\mathcal{A}, \overline{\mathcal{A}})$ является отображением моментов

$$
m:(\mathcal{A}, \overline{\mathcal{A}}, \mathbf{p}) \rightarrow \operatorname{Lie}^{*}(\mathcal{G})
$$

Условие нулевой кривизны (3.13) означает, что мы полагаем $m=0$. Это позволяет определить $\overline{\mathcal{A}}$. Обшее решение уравнения (3.13) зависит от двух полей, $\bar{A}$ и $\mu$, и имеет вид

$$
\overline{\mathcal{A}}=\left(\begin{array}{cc}
-\bar{A}+\frac{1}{2} \partial \mu-\kappa^{-1} \mu A & -\kappa^{-1} \mu \\
-\kappa^{-1} \mu \widetilde{T}-\kappa \partial\left(\bar{A}-\frac{1}{2} \partial \mu+\kappa^{-1} \mu A\right) & -\bar{A}+\kappa^{-1} \mu A-\frac{1}{2} \partial \mu
\end{array}\right)
$$

где $\bar{A}$ принимает значения в $\operatorname{Lie}(S L(N, \mathbb{C}))$. Легко проверить, что уравнение $(3.13)$ становится тождеством для $G L(2, \mathbb{C})$-матричных элементов $(1,1)$ и $(1,2)$ и совпадает с уравнениями (3.5) и (3.6) соответственно для матричных элементов $(2,1)$ и $(2,2)$.

Теперь обсудим граничные условия (3.7) и (3.8). Поскольку $m=0$, все коэффициенты из $F^{2,1}(\mathcal{A}, \overline{\mathcal{A}})$ и $F^{2,2}(\mathcal{A}, \overline{\mathcal{A}})$ в $(3.17)$ и $(3.18)$ становятся равными нулю. Выражение $\bar{\partial} \widetilde{T}$ для матричного элемента $(2,1)$ содержит члены, пропорциональные $\partial \delta\left(x_{a}\right)$. Их сокращение приводит к условию (3.8). Аналогичным образом, используя уравнение (3.18), из условия $(3.7)$ для $\bar{\partial} A$ получаем $(2,2)$-матричный элемент.

Условие нулевой кривизны этих связностей эквивалентно совместности линейной системы

$$
(\kappa \partial-\mathcal{A})\left(\begin{array}{c}
\psi \\
\kappa \partial \psi
\end{array}\right)=0, \quad(\bar{\partial}-\overline{\mathcal{A}})\left(\begin{array}{c}
\psi \\
\kappa \partial \psi
\end{array}\right)=0 .
$$

Подставляя в эту систему $\mathcal{A}(3.19)$ и $\overline{\mathcal{A}}(3.20)$, получаем линейную систему $(3.9),(3.10)$.

Редуцированная симплектическая форма получается из $(3.14),(3.19)$ и (3.20). Она совпадает с $\omega(3.11)$. 
3.3. Изомонодромные деформации. Чтобы получить нетривиальные динамические системы с гамильтонианами, модифицируем наши предыдушие процедуры. Вместо калибровочной группы $\mathcal{G}$, определенной в (3.16), рассмотрим ее параболическую подгруппу

$$
\mathcal{G}^{P}=\left\{\Sigma_{g, n} \rightarrow\left(\begin{array}{cc}
a & 0 \\
b & a^{-1}
\end{array}\right) \otimes S L(N, \mathbb{C})\right\} .
$$

Вследствие такого выбора нет ограничения моментов, обусловленного $(2,1)$-матричными элементами $G L(2, \mathbb{C})$. Таким образом, условия $(3.5)$ и $(3.8)$ в этом случае отсутствуют. Мы приходим тогда к многообразию $\mathcal{K}_{2}^{N}$ со связями $(3.6),(3.7)$ и к симплектической форме (3.11). Очевидно, что $\mathcal{W}_{2}^{N} \subset \mathcal{K}_{2}^{N} \subset \mathcal{K}_{2}$.

Рассмотрим преобразования полей в $\mathcal{K}_{2}^{N}$ вида

$$
\mu \rightarrow \mu+\xi, \quad \bar{A} \rightarrow \bar{A}+\frac{1}{\kappa} A \xi, \quad T \rightarrow T, \quad A \rightarrow A,
$$

где $\xi \in \Omega^{(-1,1)}\left(\Sigma_{g}\right)$. Они представляют собой симметрии формы $\omega(3.11)$. Преобразования (3.21) порождаются гамильтонианом

$$
\mathcal{H}_{\xi}=\int_{\Sigma_{g, n}} \xi\left(T-\frac{\left\langle A^{2}\right\rangle}{N}\right)
$$

Положим $\mathcal{H}_{\xi}=0$. Это означает, что соотношение Шугавары

$$
T=\frac{1}{N}\left\langle A^{2}\right\rangle
$$

является условием сохранения моментов относительно симметрии (3.21). Мы не фиксируем калибровку и, следовательно, получаем пространство с полями

$$
\mathcal{P}_{2}^{N}=\{A, \bar{A}, \mathbf{p}, \mu\} \sim \mathcal{K}_{2}^{N} /\left(T=\frac{1}{N}\left\langle A^{2}\right\rangle\right) .
$$

Это расслоение над пространством времен $\{\mu\}$. Как следует из (3.11), на этом пространстве форма $\omega$ принимает вид

$$
\frac{1}{2} \omega=\int_{\Sigma_{g}}\langle\delta A, \delta \bar{A}\rangle+2 \pi i \sum_{a=1}^{n} \delta\left\langle p_{a}, g_{a}^{-1} \delta g_{a}\right\rangle-\frac{1}{2 \kappa} \int_{\Sigma_{g}} \delta\left\langle A^{2}\right\rangle \delta \mu .
$$

Поскольку мы не фиксировали калибровку этого преобразования и сохранили поле $\mu$, форма $\omega(3.23)$ вырождена на $\mathcal{P}_{2}^{N}$. Если провести замену

$$
\bar{A}^{\prime}=\bar{A}-\frac{1}{\kappa} \mu A
$$

то форма (3.23) примет канонический вид

$$
\frac{1}{2} \omega=\int_{\Sigma_{g}}\left\langle\delta A, \delta \bar{A}^{\prime}\right\rangle+2 \pi i \sum_{a=1}^{n} \delta\left\langle p_{a}, g_{a}^{-1} \delta g_{a}\right\rangle
$$


Перейдем теперь к конечномерному пространству эквивалентных комплексных структур $\mathcal{T}_{g, n}$ (пространству Тейхмюллера). Касательным к пространству Тейхмюллера является пространство $H^{1}\left(\Sigma_{g}, \Gamma\right)$. Заметим, что только элементы из $H^{1}\left(\Sigma_{g}, \Gamma\right)$ вносят вклад во второй интеграл в (3.23). Согласно теореме Римана-Роха $H^{1}\left(\Sigma_{g}, \Gamma\right)$ имеет размерность

$$
l_{2}=\operatorname{dim} H^{1}\left(\Sigma_{g}, \Gamma\right)=3 g-3+n .
$$

Фиксируем точку отсчета $\mu^{0}=\left(\mu_{1}^{0}, \ldots, \mu_{l_{2}}^{0}\right)$. Тогда дифференциал

$$
\mu=\sum_{s=1}^{l_{2}} t_{s} \mu_{s}^{0}
$$

определяет новую комплексную структуру, a $\mathbf{t}=\left(t_{1}, \ldots, t_{l_{2}}\right)$ являются координатами касательного к $\mu^{0}$ вектора в $H^{1}\left(\Sigma_{g}, \Gamma\right)$.

Разлагая $\mu$ по базису (3.27), перепишем выражение для $\omega$ как

$$
\frac{1}{2} \omega=\omega^{0}-\frac{1}{\kappa} \sum_{s} \delta H_{s} \delta t_{s}, \quad H_{s}=H_{s}(A, \mathbf{t})=\frac{1}{2} \int_{\Sigma_{g}}\left\langle A^{2}\right\rangle \mu_{s}^{0},
$$

где

$$
\omega^{0}=\int_{\Sigma_{g}}\langle\delta A, \delta \bar{A}\rangle+2 \pi i \sum_{a=1}^{n} \delta\left\langle p_{a}, g_{a}^{-1} \delta g_{a}\right\rangle, \quad \mathbf{t}=\left(t_{1}, \ldots, t_{l}\right), \quad \partial_{s}=\frac{\partial}{\partial t_{s}} .
$$

Мы сохранили прежние обозначения для пространства $\mathcal{P}_{2}^{N}$ :

$$
\begin{aligned}
& \mathcal{P}_{2}^{N} \\
& \quad \downarrow \sim\{A, \bar{A}, \mathbf{p}\}=\mathcal{R} . \\
& \mathcal{T}_{g}
\end{aligned}
$$

$\mathcal{P}_{2}^{N}$ играет роль расширенного фазового пространства, в то время как $\mathcal{R}$ - стандартное фазовое пространство с невырожденной формой $\omega^{0}$. В этом случае уравнение движения для любой функции $F$ на $\mathcal{P}_{2}^{N}$ принимает вид [13]

$$
\kappa \frac{d F}{d t_{s}}=\kappa \frac{\partial F}{\partial t_{s}}+\left\{H_{s}, F\right\}_{\omega^{0}}
$$

Кроме того, имеются условия совместности для гамильтонианов (уравнения Уизема)

$$
\kappa \partial_{s} H_{r}-\kappa \partial_{r} H_{s}+\left\{H_{r}, H_{s}\right\}=0
$$

Поскольку гамильтонианы (3.28) коммутируют, сушествует тау-функиия:

$$
H_{s}=\frac{\partial}{\partial t_{s}} \log \tau
$$

На этом этапе уравнения движения тривиальны:

$$
\partial_{s} A=0, \quad \partial_{s} \bar{A}=\frac{1}{2} A \mu_{s}^{0}, \quad s=1, \ldots, l .
$$


Назовем такую систему иерархией изомонодромных деформаций. Мы обоснуем это определение ниже.

Вырожденная симплектическая форма (3.28) является инвариантной при калибровочных преобразованиях из $\widetilde{\mathcal{G}}=\operatorname{Map}\left(\Sigma_{g, n} S L(N, \mathbb{C})\right)$

$$
\begin{array}{ll}
A \rightarrow f^{-1} A f+f^{-1} \kappa \partial f, & \bar{A} \rightarrow f^{-1} \bar{A} f+f^{-1}(\bar{\partial}+\mu \partial) f, \\
p_{a} \rightarrow f^{-1}\left(x_{a}\right) p_{a} f\left(x_{a}\right), & g_{a} \rightarrow g_{a} f\left(x_{a}\right) .
\end{array}
$$

Условие нулевой кривизны

$$
(\bar{\partial}+\partial \mu) A-\kappa \partial \bar{A}+[\bar{A}, A]=0
$$

представляет собой условие нулевого момента, порожденного данной симметрией. Исходя из этого рассмотрим линейную совместную систему на $\Sigma_{g, n}$

$$
\begin{gathered}
(\kappa \partial+A) \Psi=0, \\
\left(\bar{\partial}+\sum_{s} t_{s} \mu_{s}^{0} \partial+\bar{A}\right) \Psi=0,
\end{gathered}
$$

где $\Psi \in \Omega^{0}\left(\Sigma_{g, n}, V\right)$. Матрица монодромии $Y \in \operatorname{Rep}\left(\pi_{1}\left(\Sigma_{g, n}\right)\right) \rightarrow S L(N, \mathbb{C})$ действует на решения как

$$
\Psi \rightarrow \Psi Y \text {. }
$$

Изомонодромия уравнений (3.33) и (3.34) - это независимость $Y$ от деформации комплексной структуры кривой $\Sigma_{g, n}$.

ПРЕДЛОЖЕНИЕ 3. Линейная система (3.33), (3.34) обладает свойством изомонодромных деформаций относительно "времен" $t_{s}$ тогда и только тогда, когда выполнены уравнения движения (3.30). Другими словами, уравнения (3.30) являются уравнениями, сохраняющими монодромию.

ДОКАЗАТЕЛЬСТво. Из уравнений (3.30) следует, что операторы $\partial_{s}$ коммутируют с $(\kappa \partial+A)$ и $\left(\bar{\partial}+\sum_{s} t_{s} \mu_{s}^{0} \partial+\bar{A}\right)$. Таким образом, кроме уравнений $(3.33)$ и $(3.34)$, мы имеем совместные уравнения

$$
\partial_{s} \Psi=0, \quad s=1, \ldots, l .
$$

Тогда из (3.35) следует, что

$$
\partial_{s} Y=0, \quad s=1, \ldots, l .
$$

Теперь предположим, что монодромия линейной системы (3.33), (3.34) есть условие независимости от времен (3.36). Тогда условия (3.35) удовлетворяются. Первое из уравнений движения (3.30) вытекает из системы (3.33) и (3.35), а второе - из (3.34) и (3.35).

Уравнения (3.30) не представляют большого интереса, поскольку они описывают свободное движение. Они принимают нетривиальный вид после гамильтоновой редукции относительно калибровочных преобразований (3.31). Условие нулевой кривизны (3.32) 
представляет собой уравнение моментов. Рассмотрим фиксацию калибровки и предположим, что $\bar{L}$ параметризует орбиты калибровочной группы $\widetilde{\mathcal{G}}$ в фазовом пространстве $\mathcal{R}$ :

$$
\bar{L}=f^{-1} \bar{A} f+f^{-1}(\bar{\partial}+\mu \partial) f .
$$

Это позволяет частично фиксировать $f$. В то же время калибровочное преобразование позволяет определить

$$
L=f^{-1} A f+f^{-1} \kappa \partial f .
$$

Мы сохранили те же обозначения для преобразованных матриц $p_{a}$. Подставляя эти два выражения в уравнение моментов, получаем

$$
(\bar{\partial}+\partial \mu) L-\kappa \partial \bar{L}+[\bar{L}, L]=0,
$$

где согласно (3.1) и (3.7) имеем $\left.\operatorname{Res} L\right|_{z=x_{a}}=p_{a}$. Фиксация калибровки (3.37) и уравнение моментов (3.38) оставляют только конечное число степеней свободы. Слои $\mathcal{R}^{\text {red }}=$ $\{L, \bar{L}, \mathbf{p}\}$ и расслоение $\mathcal{P}_{2}^{\text {red, } N}$ становятся конечномерными. Форма $\omega(3.28)$ на $\mathcal{P}_{2}^{\text {red, } N}$ имеет вид

$$
\omega^{0}=\int_{\Sigma_{g, n}}\langle\delta L, \delta \bar{L}\rangle+2 \pi i \sum_{a=1}^{n} \delta\left\langle p_{a}, g_{a}^{-1} \delta g_{a}\right\rangle, \quad H_{s}=H_{s}(L, \mathbf{t})=\frac{1}{2} \int_{\Sigma_{g, n}}\left\langle L^{2}\right\rangle \mu_{s}^{0}
$$

Однако система больше не является свободной, потому что согласно уравнению (3.38) $L$ зависит от $\bar{L}$ и р. Уравнения движения (3.30) принимают вид

$$
\begin{gathered}
\kappa \partial_{s} L-\kappa \partial M_{s}+\left[M_{s}, L\right]=0, \quad M_{s}=\partial_{s} f f^{-1}, \quad s=1, \ldots, l_{2}, \\
\kappa \partial_{s} \bar{L}-(\bar{\partial}+\mu \partial) M_{s}+\left[M_{s}, \bar{L}\right]=L \mu_{s}^{0} .
\end{gathered}
$$

Уравнения (3.40) аналогичны уравнениям Лакса. Основное отличие состоит в наличии дифференцирования $\partial$ по спектральному параметру. Эти уравнения нетривиальны и определяют иерархии изомонодромных деформаций, связанные с $S L(N, \mathbb{C})$-расслоениями над $\Sigma_{g, n}$. В случае $g=0,1$ они приводят к системе Шлезингера, эллиптической системе Шлезингера, мультикомпонентному обобшению уравнений Пенлеве VI [8]. Уравнения (3.41) определяют матрицу $M_{s}$. Уравнения (3.40) и (3.41) вместе с уравнением (3.38) представляют собой условия совместности линейной системы

$$
\begin{gathered}
(\kappa \partial+L) \Psi=0, \\
\left(\bar{\partial}+\sum_{s} t_{s} \mu_{s}^{0} \partial+\bar{L}\right) \Psi=0, \\
\left(\kappa \partial_{s}+M_{s}\right) \Psi=0, \quad s=1, \ldots, l_{2} .
\end{gathered}
$$

Как и в предложении 3, уравнения (3.44) обеспечивают свойство изомонодромии системы (3.42), (3.43) относительно вариаций времен $t_{s}$. 
3.4. Скейлинговый предел. Рассмотрим предел $\kappa \rightarrow 0$. Значение $\kappa=0$ называется критическим. Симплектическая форма $\omega(3.28)$ сингулярна в этом пределе. Произведем замену времен

$$
t_{s} \rightarrow t_{s}^{0}+\kappa t_{s}
$$

и возьмем времена $t_{s}^{0}\left(s=1, \ldots, l_{2}\right)$ фиксированными. После такого масштабного преобразования форма (3.28) становится регулярной. Процедура масштабного преобразования означает раздутие окрестности фиксированной точки $\mu_{s}^{(0)}$ в $\mathcal{T}_{g, n}$, и вся динамика теперь происходит в этой окрестности. Эта фиксированная точка определяется комплексными координатами

$$
w_{0}=z-\sum_{s} t_{s}^{0} \epsilon_{s}(z, \bar{z}), \quad \bar{w}_{0}=\bar{z}, \quad \partial_{\bar{w}_{0}}=\bar{\partial}+\sum_{s} t_{s}^{0} \mu_{s}^{0} .
$$

При $\kappa=0$ связность $A$ преобразуется в 1-форму $\Phi$ (поле Хиггса) $\kappa \partial+A \rightarrow \Phi$ (см. (3.31)). Пусть $L^{0}=\lim _{\kappa \rightarrow 0} L, \bar{L}^{0}=\lim _{\kappa \rightarrow 0} \bar{L}$. Тогда мы получим автономную гамильтонову систему с формой

$$
\omega^{0}=\int_{\Sigma_{g, n}}\left\langle\delta L^{0}, \delta \bar{L}^{0}\right\rangle+2 \pi i \sum_{a=1}^{n} \delta\left\langle p_{a}, g_{a}^{-1} \delta g_{a}\right\rangle
$$

и с коммутируюшими квадратичными интегралами (3.29). Фазовое пространство $\mathcal{R}^{\mathrm{red}}$ является кокасательным расслоением к пространству модулей стабильных голоморфных $S L(N, \mathbb{C})$-расслоений над $\Sigma_{g, n}$. Эти системы являются полностью интегрируемыми в случае $S L(2, \mathbb{C})$-расслоений [7].

Соответствуюшее множество линейных уравнений можно описать следующим образом. Уровень $\kappa$ можно рассматривать как постоянную Планка (см. (3.42)). Рассмотрим квазиклассический режим

$$
\Psi=\phi \exp \left(\frac{\mathcal{S}}{\kappa}\right),
$$

где $\phi$ - функция со значениями в группе, а $\mathcal{S}$ - скалярная фаза. Примем, что

$$
\frac{\partial}{\partial \bar{w}_{0}} \mathcal{S}=0, \quad \frac{\partial}{\partial t_{s}} \mathcal{S}=0
$$

В квазиклассическом пределе мы полагаем

$$
\partial \mathcal{S}=\lambda .
$$

Тогда вместо уравнений (3.42)-(3.44) получим

$$
\begin{gathered}
\left(\lambda+L^{0}\right) \Psi=0, \\
\left(\bar{\partial}_{\bar{w}_{0}}+\lambda \sum_{s} t_{s} \mu_{s}^{0}+\bar{L}^{0}\right) \Psi=0, \\
\left(\partial_{s}+M_{s}^{0}\right) \Psi=0, \quad s=1, \ldots, l_{2} .
\end{gathered}
$$

Заметим, что условия совместности для уравнений (3.46) и (3.48) представляют собой стандартные уравнения Лакса (1.1). 


\section{4. $\mathcal{W}_{3}$-СТРУКТУРЫ И ИЗОМОНОДРОМНЫЕ ДЕФОРМАЦИИ}

4.1. $\mathcal{W}_{3}$-структура. Определим пространство $\widetilde{\mathcal{W}}_{3}$ полей $(W, \rho),(T, \mu)$ на $\Sigma_{g, n}$, где $(T, \mu)$ - те же, что и в случае проективных структур, $\rho-(-2,1)$-дифференциал, а вариация $W$ является $(3,0)$-дифференциалом $[10,11]$. Будем считать, что поведение $\rho$ вблизи отмеченных точек имеет вид (ср. с (2.6))

$$
\left.\rho\right|_{z \rightarrow x_{a}} \sim\left(t_{3, a, 0}+t_{3, a, 1}\left(z-x_{a}^{0}\right)\right) \bar{\partial} \chi_{a}(z, \bar{z}) .
$$

Дуальное поле $W$ имеет полюсы в отмеченных точках:

$$
\left.W\right|_{z \rightarrow x_{a}} \sim \frac{W_{-3, a}}{\left(z-x_{a}\right)^{3}}+\frac{W_{-2, a}}{\left(z-x_{a}\right)^{2}}+\frac{W_{-1, a}}{\left(z-x_{a}\right)}+\cdots .
$$

ОПРЕДЕЛЕНИЕ 3 . Пространство $\mathcal{W}_{3}$-структур на $\Sigma_{g, n}$ является подмножеством полей в $\widetilde{\mathcal{W}}_{3}$, которые удовлетворяют уравнениям

$$
\begin{aligned}
-2 \kappa^{2} \partial^{3} \mu+(\bar{\partial}+\mu \partial+2 \partial \mu) T & =\kappa^{2} \partial^{2}\left(\partial^{2}-\frac{1}{\kappa^{2}} T\right) \rho-\frac{2}{\kappa} \partial(W \rho)-\frac{1}{\kappa} W \partial \rho \\
\left(\bar{\partial}+\rho \partial^{2}+(\mu+2 \partial \rho) \partial+3 \partial \mu\right) W & =\kappa^{3}\left(\partial^{2}-\frac{1}{\kappa^{2}} T\right) \partial\left(\partial \mu-\frac{2}{3}\left(\partial^{2}-\frac{1}{\kappa^{2}} T\right) \rho\right)
\end{aligned}
$$

с фиксированными значениями

$$
\mathbf{T}_{2}=\left(T_{-2,1}, \ldots, T_{-2, n}\right)
$$

в (2.8) и с фиксированными значениями

$$
\mathbf{W}_{2}=\left(W_{-3,1}, \ldots, W_{-3, n}\right)
$$

в $(4.2)$.

ПрЕДЛОЖЕНИЕ 4. Уравнения (4.3), (4.4) представляют собой условия совместности линейной системь

$$
\begin{gathered}
\left(\kappa^{3} \partial^{3}+\kappa T \partial+W\right) \Psi=0 \\
\left(\bar{\partial}+\frac{2}{3}\left(\partial^{2}-\frac{1}{\kappa^{2}} T\right) \rho-\partial \mu+(\mu-\partial \rho) \partial-\rho \partial^{2}\right) \Psi=0
\end{gathered}
$$

где $\Psi$ - сечение расслоения $\Omega^{(-1,0)}\left(\Sigma_{g, n}\right)$.

Пространство $\mathcal{W}_{3}$ можно снабдить симплектической формой

$$
\omega=\int_{\Sigma_{g, n}}(\delta T \delta \mu+\delta W \delta \rho) .
$$

Как и для проективных структур, пространство $\mathcal{W}_{3}$ можно получить из пространства $\mathcal{N}_{3}$ плоских $S L(3, \mathbb{C})$-связностей

$$
\mathcal{N}_{3}=\{a, \bar{a} \mid F(a, \bar{a})=0\}
$$


над $\Sigma_{g, n}$ с симплектической формой (2.16). На самом деле, представление (4.9) следует из (2.16). Условие нулевой кривизны порождает калибровочные симметрии на $\mathcal{N}_{3}$. Будем считать, что матричные элементы алгебры Ли калибровочной группы непрерывны в отмеченных точках, более того,

$$
\left.x^{13}\right|_{z \rightarrow x_{a}}=O\left(z-x_{a}\right)^{2},\left.\quad x^{23}\right|_{z \rightarrow x_{a}}=O\left(z-x_{a}\right)
$$

(см. (2.17)). В действительности условие (4.10) определяет подалгебру в алгебре Ли непрерывных калибровочных преобразований.

Частичная фиксация калибровки относительно параболической подгруппы [10]

$$
P=\left(\begin{array}{lll}
* & * & 0 \\
* & * & 0 \\
* & * & *
\end{array}\right)
$$

позволяет подобрать специальную форму связностей

$$
a=\left(\begin{array}{ccc}
0 & 1 & 0 \\
0 & 0 & 1 \\
W & T & 0
\end{array}\right)
$$

при которой $W$ и $T$ ведут себя предписанным образом в окрестностях отмеченных точек. Точный вид матришы $\bar{a}$ и уравнений (4.3) и (4.4) получаются из условия нулевой кривизны. Определяюшие свойства (4.10) калибровочной алгебры позволяют фиксировать коэффициенты при старших полюсах (4.5) и (4.6). Они определяют значение отображения моментов $m=F(a, \bar{a})$. Ниже мы обобщим эту процедуру.

Условие нулевой кривизны представляет собой условие совместности для линейной системы

$$
(\kappa \partial-a)\left(\begin{array}{c}
\psi \\
\kappa \partial \psi \\
\kappa^{2} \partial^{2} \psi
\end{array}\right)=0, \quad(\bar{\partial}-\bar{a})\left(\begin{array}{c}
\psi \\
\kappa \partial \psi \\
\kappa^{2} \partial^{2} \psi
\end{array}\right)=0
$$

где $\psi \in \Omega^{(-1,0)}\left(\Sigma_{g, n}\right)$. Для специального вида $a(4.12)$ и $\bar{a}$ эта система сводится к линейной системе (4.7), (4.8).

4.2. $\mathcal{W}_{3}^{N}$-структуры. Как и в п. 3.1 рассмотрим $S L(N, \mathbb{C})$-расслоение $V$ над $\Sigma_{g, n}$ с фиксированной комплексной структурой. Пусть $(A, \bar{A})-$ связности в $V$. Обобшим $\mathcal{W}_{3}$-структуру (4.3), (4.4), учитывая калибровочные степени свободы.

Определим пространство

$$
\widetilde{\mathcal{W}}_{3}^{N}=\widetilde{\mathcal{W}}_{2}^{N} \cup(W, \rho),
$$

где $\rho$ и $W$ удовлетворяют условиям (4.1) и (4.2), и введем матрицы

$$
\begin{aligned}
\widetilde{T} & =T-3\left(A^{2}+\kappa \partial A\right), \\
\widetilde{W} & =W+T A-A^{3}-\kappa\left(A \partial A+\partial A^{2}\right)-\kappa^{2} \partial^{2} A .
\end{aligned}
$$


ОпРЕДЕЛЕниЕ $4 . \mathcal{W}_{3}^{N}$-структура на $\Sigma_{g, n}$ является подмножеством полей в $\widetilde{\mathcal{W}}_{3}^{N}$, удовлетворяюших равенствам

$$
\begin{gathered}
\bar{\partial} \widetilde{W}-\kappa \partial f_{5}+\widetilde{W} f_{1}+\widetilde{T} f_{3}-3 A f_{5}-f_{7} \widetilde{W}=0, \\
\bar{\partial} \widetilde{T}-\kappa \partial f_{6}+\widetilde{W} f_{2}+\widetilde{T} f_{4}-3 A f_{6}-f_{7} \widetilde{T}-f_{5}=0, \\
\bar{\partial} A-\kappa \partial \bar{A}+[\bar{A}, A]=0, \\
A_{-1, a}=p_{a} \\
T_{-2, a}=\frac{3\left\langle\left(p_{a}^{0}\right)^{2}\right\rangle}{N}, \\
W_{-3, a}=\frac{\left\langle\left(p_{a}^{0}\right)^{3}\right\rangle-3 \kappa\left\langle\left(p_{a}^{0}\right)^{2}\right\rangle}{N}, a=1, \ldots, n .
\end{gathered}
$$

Матричные коэффициенты $f_{j}$ имеют вид

$$
\begin{aligned}
f_{1}= & -\frac{2}{3}\left(\partial^{2}-\frac{1}{\kappa^{2}} T\right) \rho+\partial \mu-\bar{A}+\frac{2}{\kappa} A \partial \rho-\frac{1}{\kappa} \mu A-\frac{2}{\kappa^{2}} A^{2} \rho, \\
f_{2}= & -\frac{1}{\kappa}(\mu-\partial \rho)-\frac{3}{\kappa^{2}} A \rho, \\
f_{3}= & \kappa \partial f_{1}-\frac{1}{\kappa^{2}} \widetilde{W} \rho= \\
= & -\frac{2}{3} \kappa \partial\left(\partial^{2}-\frac{1}{\kappa^{2}} T\right) \rho+\kappa \partial^{2} \mu-\kappa \partial \bar{A}+2 \partial(A \partial \rho)- \\
& -\frac{1}{\kappa^{2}} \widetilde{W} \rho-\partial\left(\mu A+\frac{2}{\kappa} A^{2} \rho\right), \\
f_{4}= & \kappa \partial f_{2}+f_{1}-\frac{1}{\kappa^{2}} \widetilde{T} \rho= \\
= & \frac{1}{3}\left(\partial^{2}-\frac{1}{\kappa^{2}} T\right) \rho-\frac{1}{\kappa} A \partial \rho-\bar{A}+\frac{1}{\kappa^{2}} A^{2} \rho-\frac{1}{\kappa} \mu A, \\
f_{5}= & -\frac{\mu}{\kappa} \widetilde{W}+\kappa \partial f_{3}= \\
= & -\frac{2}{3} \kappa^{2} \partial^{2}\left(\partial^{2}-\frac{1}{\kappa^{2}} T\right) \rho+\kappa^{2} \partial^{3} \mu-\kappa^{2} \partial^{2} \bar{A}+2 \kappa \partial^{2}(A \partial \rho)- \\
& -\frac{1}{\kappa} \partial(\widetilde{W} \rho)-\frac{\mu}{\kappa} \widetilde{W}-\kappa \partial^{2}\left(\mu A+\frac{2}{\kappa} A^{2} \rho\right), \\
f_{6}= & -\frac{\mu}{\kappa} \widetilde{T}+\kappa \partial f_{4}+f_{3}= \\
= & -\frac{1}{3} \kappa \partial\left(\partial^{2}-\frac{1}{\kappa^{2}} T\right) \rho+\kappa \partial^{2} \mu-\kappa \partial \bar{A}+\partial(A \partial \rho)- \\
& -\frac{1}{\kappa^{2}} \widetilde{W} \rho-\frac{\mu}{\kappa} \widetilde{T}+\frac{3}{\kappa} \partial\left(A^{2}\right) \rho-2 \partial\left(\mu A+\frac{2}{\kappa} A^{2} \rho\right), \\
f_{7}= & \frac{3}{\kappa} \mu A-\partial \mu+f_{4}= \\
= & \frac{2}{\kappa} \mu A-\partial \mu+\frac{1}{3}\left(\partial^{2}-\frac{1}{\kappa^{2}} T\right) \rho-\frac{1}{\kappa} A \partial \rho-\bar{A}+\frac{1}{\kappa^{2}} A^{2} \rho . \\
\mu &
\end{aligned}
$$


Заметим, что уравнения (4.15) и (4.16) сводятся к стандартной $\mathcal{W}_{3}$-структуре, если $A=$ $\bar{A}=0$, а уравнение (4.17) представляет собой условие нулевой кривизны расслоения $V$.

ПРЕДЛОЖЕнИЕ 5. Уравнения (4.15)-(4.17) представ.яяют собой условия совместности для линейной системь

$$
\begin{aligned}
& \left(\kappa^{3} \partial^{3}+3 A \kappa^{2} \partial^{2}+\kappa \widetilde{T} \partial+\widetilde{W}\right) \Psi=0, \\
& \left(\bar{\partial}-f_{1}-f_{2} \kappa \partial-\rho \partial^{2}\right) \Psi=0,
\end{aligned}
$$

где $\Psi$ - сечение расслоения $\Omega^{(-1,0)}\left(\Sigma_{g, n}\right.$, Aut $\left.V\right)$.

Как и ранше, $\mathcal{W}_{3}^{N}$ имеет естественную симплектическую форму

$$
\omega=\int_{\Sigma_{g, n}}\left[-\frac{N}{\kappa} \delta T \delta \mu-\frac{N}{\kappa^{2}} \delta W \delta \rho+3\langle\delta A, \delta \bar{A}\rangle\right]+6 \pi i \sum_{a=1}^{n} \delta\left\langle p_{a}, g_{a}^{-1} \delta g_{a}\right\rangle
$$

ДоКАЗАТЕЛЬСТВО предложения 5 и вывод формулы (4.30) основаны на той же процедуре, которую мы использовали ранее для $\mathcal{W}_{2}^{N}$. Рассмотрим связности $\kappa \partial+\mathcal{A}$ и $\bar{\partial}+\overline{\mathcal{A}}$ в векторном расслоении $(S(G L(3, \mathbb{C}) \otimes G L(N, \mathbb{C})))$. Первая компонента $(\mathcal{A}, \overline{\mathcal{A}})$ действует на сечениях 3 -струй расслоения $\Omega^{(-1,0)}\left(\Sigma_{g, n}\right)$, а вторая - на сечениях расслоения $\Omega^{(-1,0)}\left(\Sigma_{g, n}\right.$, Aut $\left.V\right)$. Определим пространство $\mathcal{K}_{3}$ связностей $(\mathcal{A}, \overline{\mathcal{A}})$ и множество коприсоединенных орбит (3.2):

$$
\left(\mathcal{A}, \overline{\mathcal{A}}, \mathbf{1}_{3} \otimes \mathbf{p}\right) .
$$

Предположим, что $\mathcal{A}$ удовлетворяет дополнительным ограничениям:

а) $\mathcal{A}$-компонента калибровочно-эквивалентна специальной форме

$$
\mathcal{A} \sim D S \otimes \mathbf{1}_{N}+\mathbf{1}_{3} \otimes(-A)
$$

где

$$
D S=\left(\begin{array}{ccc}
0 & 1 & 0 \\
0 & 0 & 1 \\
W & T & 0
\end{array}\right) ;
$$

б) вблизи отмеченных точек $W$ имеет вид (4.2);

в) вблизи отмеченных точек $T$ имеет вид (2.8);

г) вблизи отмеченных точек $A$ имеет вид (3.1).

Предложение 5 вытекает из следуюшего утверждения.

ПРЕДЛОЖЕНИЕ $6 . \mathcal{W}_{3}^{N}$ является подмножеством в $\mathcal{K}_{3}$, удовлетворяющим условию нулевой кривизны

$$
\bar{\partial} \mathcal{A}-\kappa \partial \overline{\mathcal{A}}+[\mathcal{A}, \overline{\mathcal{A}}]=0 .
$$


ДокАЗАТЕльСТво. Симплектическая форма $\omega^{\prime}$ на $\mathcal{K}_{3}$ аналогична симплектической форме на $\mathcal{K}_{2}$ :

$$
\omega^{\prime}=\int_{\Sigma_{g, n}}\langle\langle\delta \mathcal{A}, \delta \overline{\mathcal{A}}\rangle\rangle+6 \pi i \sum_{a=1}^{n} \delta\left\langle p_{a}, g_{a}^{-1} \delta g_{a}\right\rangle
$$

Калибровочная группа симметрии симплектической формы $\omega^{\prime}$ имеет вид

$$
\mathcal{G}=\left\{\operatorname{Map}\left(\Sigma_{g, n} \rightarrow S L\left(3 N^{2}, \mathbb{C}\right)\right)\right\} .
$$

Действие группы $\mathcal{G}$ такое же, как и в (3.16). Алгебра $\operatorname{Lie}(\mathcal{G})$ определяется тем, что матричные элементы непрерывны в отмеченных точках с дополнительными ограничениями на элементы $x_{\alpha \beta}^{13}, x_{\alpha \beta}^{23}$ :

$$
\begin{aligned}
\left.\operatorname{tr}\right|_{V_{N}} x^{13} & \sim O\left(z-x_{a}\right)^{2}, & & x_{\alpha \beta}^{13} \sim O\left(z-x_{a}\right)^{3}, \\
\left.\operatorname{tr}\right|_{V_{N}} x^{23} \alpha \beta & \sim O\left(z-x_{a}\right), & & x_{\alpha \beta}^{23} \sim O\left(z-x_{a}\right)^{2} .
\end{aligned}
$$

Для элементов дуального пространства $y \in \operatorname{Lie}^{*}(\mathcal{G})$ имеем

$$
\begin{array}{rlrl}
\left.\operatorname{tr}\right|_{V_{N}} y^{31} & \sim \sum_{k \geqslant 2} b_{k} \partial^{k} \delta\left(x_{a}\right), & y_{\alpha \beta}^{31} & \sim \sum_{k \geqslant 3} b_{k, \alpha, \beta} \partial^{k} \delta\left(x_{a}\right), \\
\left.\operatorname{tr}\right|_{V_{N}} y^{32} & \sim \sum_{k \geqslant 1} b_{k} \partial^{k} \delta\left(x_{a}\right), & y_{\alpha \beta}^{32} & \sim \sum_{k \geqslant 2} b_{k, \alpha, \beta} \partial^{k} \delta\left(x_{a}\right), \\
y_{\alpha \beta}^{J K} & \sim \sum_{k \geqslant 0} b_{k, \alpha, \beta} \partial^{k} \delta\left(x_{a}\right), & J \neq 3, \quad K \neq 1,2 .
\end{array}
$$

Выберем $\mathcal{A}$ в виде

$$
\mathcal{A}=\left(\begin{array}{ccc}
0 & E & 0 \\
0 & 0 & E \\
\widetilde{W} & \widetilde{T} & -3 A
\end{array}\right) .
$$

Подставляя (4.37) в уравнение (4.32), находим $\overline{\mathcal{A}}$. Решения $\overline{\mathcal{A}}$ параметризуются полями $\mu$ и $\rho$, а также матрищей $\bar{A}$. Имеем

$$
\overline{\mathcal{A}}=\left(\begin{array}{ccc}
f_{1} & f_{2} & -\rho / \kappa^{2} \\
f_{3} & f_{4} & -\mu / \kappa \\
f_{5} & f_{6} & f_{7}
\end{array}\right) .
$$

Положим $f_{1}+f_{4}+f_{7}=-3 \bar{A}$. Это условие вместе с алгебраическим уравнением (4.32) для $(J, K)$-блоков, $J=1,2, K=1,2,3$, позволяет найти $f_{1}, \ldots, f_{7}$ в (4.21)-(4.27). Из дифференциального тождества, появляюшегося в последней строке (при $J=3, K=$ $1,2,3)$, получаем уравнения (4.15)-(4.17). Поведение наиболее сингулярных членов вблизи отмеченных точек (см. (4.18)-(4.20)) определяется специальным видом элементов из $\operatorname{Lie}^{*}(\mathcal{G})(4.34)-(4.36)$.

Более того, из формы $\omega^{\prime}(4.33)$ получается форма $\omega(4.30)$ на пространстве $\mathcal{W}_{3}^{N}$.

Вследствие специального вида связностей $\mathcal{A}$ и $\overline{\mathcal{A}}$ совместная система дифференциальных уравнений

$$
(\kappa \partial-\mathcal{A})\left(\begin{array}{c}
\Psi \\
\kappa \partial \Psi \\
\kappa^{2} \partial^{2} \Psi
\end{array}\right)=0, \quad(\bar{\partial}-\overline{\mathcal{A}})\left(\begin{array}{c}
\Psi \\
\kappa \partial \Psi \\
\kappa^{2} \partial^{2} \Psi
\end{array}\right)=0
$$

эквивалентна системе (4.28), (4.29). 
4.3. $\mathcal{W}_{3}^{N}$-структуры и изомонодромные деформации. Чтобы получить нетривиальный фазовый поток, необходимо избавиться от ограничительных связей (4.15), $(4.16),(4.19)$ и $(4.20)$. Оставшиеся связи порождают калибровочную подгруппу

$$
\mathcal{G}^{P}=\left\{\operatorname{Map}\left(\Sigma_{g, n} \rightarrow P\right)\right\}
$$

где $P$ - параболическая подгруппа (4.11). Таким образом, мы приходим к пространству $\mathcal{K}_{3}^{N} \subset \mathcal{K}_{3}$ полей $\left(W, \rho, T, \mu, A, \bar{A}, p_{a}\right)$ со связями (4.17) и (4.18).

Форма $\omega(4.30)$, будучи ограничена на $\mathcal{K}_{3}^{N}$, инвариантна при преобразованиях

$$
A \rightarrow A, \quad \bar{A} \rightarrow \bar{A}+\frac{1}{\kappa} \xi_{2} A+\frac{1}{\kappa^{2}} \xi_{3} A^{2}, \quad T \rightarrow T, \quad \mu \rightarrow \mu+\xi_{2}, \quad \rho \rightarrow \rho+\xi_{3},
$$

где $\xi_{j} \in \Omega^{(1-j, 1)}\left(\Sigma_{g, n}\right)$, с тем же поведением вблизи отмеченных точек, что и для $\mu$ и $\rho$. Условия на отображение моментов, порождаемые этими преобразованиями, имеют вид

$$
T=\frac{3}{2 N}\left\langle A^{2}\right\rangle, \quad W=\frac{1}{N}\left\langle A^{3}\right\rangle .
$$

Подставляя $T$ и $W$ в выражение для формы $\omega(4.30)$, получаем

$$
\begin{aligned}
\frac{1}{3} \omega= & \int_{\Sigma_{g, n}}\langle\delta A, \delta \bar{A}\rangle+2 \pi i \sum_{a=1}^{n} \delta\left\langle p_{a}, g_{a}^{-1} \delta g_{a}\right\rangle- \\
& -\frac{1}{\kappa} \int_{\Sigma_{g, n}}\langle\delta A, A\rangle \delta \mu-\frac{1}{\kappa^{2}} \int_{\Sigma_{g, n}}\left\langle\delta A, A^{2}\right\rangle \delta \rho .
\end{aligned}
$$

Форма $\omega$ становится вырожденной, поскольку мы не фиксировали калибровку относительно преобразований (4.38). Если произвести замену

$$
\bar{A} \rightarrow \bar{A}^{\prime}=\bar{A}-\frac{1}{\kappa} \mu A-\frac{1}{2 \kappa^{2}} \rho\left(A^{2}+\kappa \partial A\right),
$$

то форма $\omega$ примет канонический вид

$$
\frac{1}{3} \omega=\int_{\Sigma_{g, n}}\left\langle\delta A, \delta \bar{A}^{\prime}\right\rangle+2 \pi i \sum_{a=1}^{n} \delta\left\langle p_{a}, g_{a}^{-1} \delta g_{a}\right\rangle .
$$

Она инвариантна при калибровочных преобразованиях

$$
A \rightarrow f A f^{-1}+f \kappa \partial f^{-1}, \quad \bar{A}^{\prime} \rightarrow f \bar{A}^{\prime} f^{-1}+f \bar{\partial} f^{-1} .
$$

Уравнение моментов, следуюшее из этой симметрии, имеет вид

$$
F\left(A, \bar{A}^{\prime}\right):=\bar{\partial} A-\kappa \partial \bar{A}+[\bar{A}, A]=0,
$$

или в исходных переменных

$$
\bar{\partial} A+\partial(\mu A)+\frac{1}{2} \partial(\rho \partial A)+\frac{1}{2 \kappa}\left(2 \rho A \partial A+\partial \rho A^{2}\right)-\kappa \partial \bar{A}+[\bar{A}, A]=0 .
$$


В этом случае связи становятся нелинейными.

Теперь вместо бесконечномерного пространства гладких дифференциалов $\rho$ рассмотрим конечномерное пространство $H^{1}\left(\Sigma_{g, n}, \Gamma^{2}\right)$. Оно имеет размерность

$$
l_{3}=\operatorname{dim} H^{1}\left(\Sigma_{g, n}, \Gamma^{2}\right)=5 g-5+2 n .
$$

Разлагая $\rho$ по базису в $H^{1}\left(\Sigma_{g, n}, \Gamma^{2}\right)$, имеем

$$
\rho=\sum_{s=1}^{l_{3}} t_{3, s} \rho_{s}^{0}
$$

Тогда из (4.39) получаем

$$
\begin{aligned}
\frac{1}{3} \omega & =\omega^{0}-\frac{1}{\kappa} \sum_{s=1}^{l_{2}} \delta H_{2, s} \delta t_{2, s}-\frac{1}{2 \kappa^{2}} \sum_{s=1}^{l_{3}} \delta H_{3, s} \delta t_{3, s}, \\
H_{2, s} & =\frac{1}{2} \int_{\Sigma_{g, n}}\left\langle A^{2}\right\rangle \mu_{s}^{(0)}, \quad H_{3, s}=\frac{1}{3} \int_{\Sigma_{g, n}}\left\langle A^{3}\right\rangle \rho_{s}^{(0)},
\end{aligned}
$$

где

$$
\omega^{0}=\int_{\Sigma_{g, n}}\left\langle\delta A, \delta \bar{A}-\frac{\rho}{2 \kappa} \delta \partial A\right\rangle+2 \pi i \sum_{a=1}^{n} \delta\left\langle p_{a}, g_{a}^{-1} \delta g_{a}\right\rangle .
$$

Уравнения движения, определяемые формой $\omega$, имеют вид

$$
\begin{gathered}
\partial_{r} A=0, \quad r=(k, s), \quad k=2,3, \quad \partial_{r}=\frac{\partial}{\partial t_{k, s}} \\
\partial_{r} \bar{A}^{\prime}=0 .
\end{gathered}
$$

Решения описывают свободное движение:

$$
\bar{A}=\bar{A}_{0}+\frac{1}{\kappa} A_{0} \sum_{s} \mu_{s}^{(0)} t_{2, s}+\frac{1}{2 \kappa^{2}}\left(A_{0}^{2}+\kappa \partial A_{0}\right) \sum_{s} \rho_{s}^{(0)} t_{3, s}
$$

Используя калибровочную симметрию, представим поля в виде

$$
L=f A f^{-1}+f \kappa \partial f^{-1}, \quad \bar{L}^{\prime}=f \bar{A}^{\prime} f^{-1}+f \bar{\partial} f^{-1},
$$

где $M_{r}=f^{-1} \partial_{r} f$. Таким образом, мы получили конечномерное расслоение $\mathcal{P}_{3}^{\text {red, } N}=$ $\{L, \bar{L}, \mathbf{p}, \mu, \rho\}$. Расслоение $\mathcal{P}_{3}^{N}$ имеет те же слои $\mathcal{R}^{\text {red }}$, что и расслоение $\mathcal{P}_{2}^{\text {red, } N}$.

Уравнение (4.41) принимает вид

$$
\begin{gathered}
\bar{\partial} L+\partial(\mu L)+\frac{1}{2} \partial(\rho \partial L)+\frac{1}{2 \kappa}\left(2 \rho L \partial L+\partial \rho L^{2}\right)-\kappa \partial \bar{L}+[\bar{L}, L]=0 \\
\left.L\right|_{z \rightarrow x_{a}}=\frac{p_{a}}{z-x_{a}}
\end{gathered}
$$

где

$$
\bar{L}=\bar{L}^{\prime}+\frac{1}{\kappa} \mu L+\frac{1}{2 \kappa^{2}} \rho\left(L^{2}+\kappa \partial L\right) .
$$


На редуцированном фазовом пространстве $\left(L, \bar{L}^{\prime}\right)$ уравнение движения (4.44) принимает вид уравнения Лакса:

$$
\partial_{r} L-\kappa \partial M_{r}+\left[M_{r}, L\right]=0 .
$$

Уравнение (4.45) позволяет определить матрицы $M_{r}$ в уравнении Лакса:

$$
\partial_{r} \bar{L}^{\prime}-\bar{\partial} M_{r}+\left[M_{r}, \bar{L}^{\prime}\right]=0 .
$$

Уравнения (4.48) и (4.49) с условием нулевой кривизны (4.47) представляют собой условие совместности линейной системы

$$
\begin{aligned}
(\kappa \partial+L) \Psi & =0, \\
\left(\bar{\partial}+\bar{L}^{\prime}\right) \Psi & =0, \\
\left(\kappa \partial_{r}+M_{r}\right) \Psi & =0 .
\end{aligned}
$$

Последнее уравнение означает, что матрица монодромии $Y$ линейной системы (4.50), (4.51) на $\Sigma_{g, n}$ не зависит от $\mathcal{W}_{3}$-модулей $\mu_{r}$ и $\rho_{r}$.

На критическом уровне $\kappa=0$ системы переходят в системы Хитчина с квадратичными и кубичными коммутируюшими интегралами.

Рассмотрим рациональную кривую $\Sigma_{0, n} \mathrm{c} n$ фиксированными отмеченными точками $x_{1}^{0}, \ldots, x_{n}^{0}$. Согласно $(3.26)$ и (4.42) мы имеем $8 n-8$ времен. Поскольку в этом случае $\bar{L}=0$, уравнение (4.47) принимает вид

$$
\begin{aligned}
{[\bar{\partial}+} & \left.\partial \sum_{a=1}^{n} t_{2, a} \bar{\partial} \chi_{a}(z, \bar{z})+\frac{1}{2 \kappa} \partial \sum_{a=1}^{n}\left(t_{3, a, 0}+t_{3, a, 1}\left(z-x_{a}^{0}\right)\right) \bar{\partial} \chi_{a}(z, \bar{z}) L\right] L+ \\
& +\frac{1}{2} \partial \sum_{a=1}^{n}\left(t_{3, a, 0}+t_{3, a, 1}\left(z-x_{a}^{0}\right)\right) \bar{\partial} \chi_{a}(z, \bar{z}) \partial L+ \\
& +\frac{1}{\kappa} \sum_{a=1}^{n}\left(t_{3, a, 0}+t_{3, a, 1}\left(z-x_{a}^{0}\right)\right) \bar{\partial} \bar{\chi}_{a}(z, \bar{z}) L \partial L=0 .
\end{aligned}
$$

Напомним, что времена $t_{2, a}$ связаны с положениями отмеченных точек $\left(t_{2, a}=x_{a}-x_{a}^{0}\right)$. Если $t_{3, a}=0$, то решение этого уравнения

$$
L=\sum_{a=1}^{n} \frac{p_{a}}{w-x_{a}}, \quad w=z-\sum_{a=1}^{n} t_{2, a} \chi_{a}(z, \bar{z})
$$

представляет собой $L$-оператор для системы Шлезингера. В общем случае $\left(t_{3, a} \neq 0\right)$ уравнение является нелинейным и его решения неизвестны. 


\section{5. ЗАКЛЮЧЕНИЕ}

Обобшение предыдушего анализа на произвольные $\mathcal{W}_{k}^{N}$-структуры осушествляется непосредственно, хотя явные вычисления оказываются достаточно громоздкими. В данном разделе мы приведем некоторые обшие формулы.

Пусть $W_{j}$ - множество $j$-дифференциалов на $\Sigma_{g, n}, \rho_{j}$ - дуальные объекты, $j=2, \ldots$ $\ldots, k, W_{2}=T, \rho_{2}=\mu$. Мы имеем также калибровочные данные $(A, \bar{A})$ и коприсоединенные орбиты в отмеченных точках $p_{a}, a=1, \ldots, n$. Примем, что $W_{j}, \rho_{j}$ ведут себя вблизи отмеченных точек как

$$
\begin{aligned}
\left.W_{j}\right|_{z \rightarrow x_{a}} & \sim \frac{W_{-j, a}}{\left(z-x_{a}\right)^{j}}+\frac{W_{-j+1, a}}{\left(z-x_{a}\right)^{j-1}}+\cdots, \\
\left.\rho_{j}\right|_{z \rightarrow x_{a}} & \sim\left(t_{j, a, 0}+t_{j, a, 1}\left(z-x_{a}^{0}\right)+\cdots+t_{j, a, j-2}\left(z-x_{a}\right)^{j-2}\right) \bar{\partial} \chi_{a}(z, \bar{z})
\end{aligned}
$$

и $A$ имеет полюсы первого порядка (3.1) с вычетами (3.7). Коэффициенты $W_{-j, a}$ старшего порядка являются линейными комбинациями соответствуюших элементов Казимира до $j$-го порядка:

$$
W_{-j, a} \sim \frac{1}{N}\left(\left\langle\left(p_{a}^{0}\right)^{j}\right\rangle+\cdots\right) .
$$

Пусть $\Psi$ - сечение расслоения $\Omega^{(-(k-1) / 2,0)}\left(\Sigma_{g, n}, \operatorname{Aut} V\right)$. Тогда $\mathcal{W}_{k}^{N}$-структура на $\Sigma_{g, n}$ является множеством полей

$$
\left(W_{j}, \rho_{j}\right), \quad j=2, \ldots, k, \quad(A, \bar{A}), \quad p_{a}, \quad a=1, \ldots, n,
$$

при условии, что линейная система

$$
\begin{aligned}
& \left(\kappa^{k} \partial^{k}+k A \kappa^{k-1} \partial^{k-1}+\cdots+\widetilde{W}_{k}\right) \Psi=0, \\
& \left(\bar{\partial}+\alpha_{k} \partial^{k-1}+\cdots+\alpha_{1}\right) \Psi=0
\end{aligned}
$$

совместна. Здесь

$$
\widetilde{W}_{k}=W_{k}+A W_{k-1}+A^{2} W_{k-2}+\cdots+A^{k-2} W_{2}-A^{k},
$$

а другие коэффициенты определяются из условия того, что связности $\mathcal{A}, \overline{\mathcal{A}}$ в $S L(k N, \mathbb{C})-$ расслоении с

$$
\begin{aligned}
\mathcal{A} & =D S \otimes \mathbf{I}_{N}+\mathbf{I}_{k} \otimes(-A) \\
D S & =\left(\begin{array}{cccccc}
0 & 1 & 0 & \ldots & 0 & 0 \\
0 & 0 & 1 & \ldots & 0 & 0 \\
\ldots \ldots \ldots \ldots \ldots \ldots \ldots \ldots \ldots \ldots & \ldots \ldots \\
0 & 0 & 0 & \ldots & 0 & 1 \\
W_{k} & W_{k-1} & W_{k-2} & \ldots & W_{2} & 0
\end{array}\right)
\end{aligned}
$$

плоские. Симплектическая форма на $\mathcal{W}_{k}^{N}$ имеет универсальную структуру:

$$
\omega=\int_{\Sigma_{g, n}}\left[k\langle\delta A, \delta \bar{A}\rangle-N \sum_{j=2}^{k} \frac{1}{\kappa^{j-1}} \delta W_{j} \delta \rho_{j}\right]+2 k \pi i \sum_{a=1}^{n} \delta\left\langle p_{a}, g_{a}^{-1} \delta g_{a}\right\rangle .
$$


Мы опускаем детали вычислений, которые в основном подобны тем, которые проводились в случае $\mathcal{W}_{3}^{N}$.

Как и раньше, связи на поля $W_{j}, \rho_{j}, j=2, \ldots, k$, могут быть опушены при ограничении на параболическую подгруппу группы калибровочных преобразований. Форма допускает абелевы симметрии

$$
\rho_{j} \rightarrow \rho_{j}+\xi_{j} \quad(j=2, \ldots, k), \quad \bar{A} \rightarrow \bar{A}+\sum_{j=2}^{k} \frac{\left(A^{j-1}+\cdots\right) \xi_{j}}{\kappa^{j-1}}
$$

где $\xi_{j} \in \Omega^{(-j+1,1)}\left(\Sigma_{g, n}\right)$, и мы опускаем члены, содержашие производные по $z$. Эти симметрии порождаются связями

$$
W_{j}=\frac{k\left\langle A^{j}+\cdots\right\rangle}{N j}
$$

Подставляя (5.2) в (5.1), получаем симплектическую форму

$$
\frac{1}{k} \omega=\int_{\Sigma_{g, n}}\langle\delta A, \delta \bar{A}+\cdots\rangle+2 \pi i \sum_{a=1}^{n} \delta\left\langle p_{a}, g_{a}^{-1} \delta g_{a}\right\rangle-\sum_{j=2}^{k} \frac{1}{\kappa^{j-1}} \int_{\Sigma_{g, n}}\left\langle A^{j-1} \delta A\right\rangle \delta \rho_{j}
$$

Ограничим поля $\rho_{j}$ на пространство $H^{1}\left(\Sigma_{g, n}, \Gamma^{j-1}\right)$. Пусть

$$
\rho_{j}=\sum_{s=1}^{l_{j}} t_{j, s} \rho_{j, s}^{0} \quad\left(l_{j}=\operatorname{dim} H^{1}\left(\Sigma_{g, n}, \Gamma^{j-1}\right)=(2 j-1)(g-1)+(j-1) n\right)
$$

- разложение $\rho_{j}$ по базису в $H^{1}\left(\Sigma_{g, n}, \Gamma^{j-1}\right)$. Тогда мы приходим к форме

$$
\frac{1}{k} \omega=\omega^{0}-\sum_{j=2}^{k} \frac{1}{\kappa^{j-1}} \sum_{s=1}^{l_{j}} \delta H_{j, s} \delta t_{j, s}
$$

где

$$
\omega^{0}=\int_{\Sigma_{g, n}}\langle\delta A, \delta \bar{A}+\cdots\rangle+2 \pi i \sum_{a=1}^{n} \delta\left\langle p_{a}, g_{a}^{-1} \delta g_{a}\right\rangle, \quad H_{j, s}=\frac{1}{j} \int_{\Sigma_{g, n}}\left\langle A^{j}\right\rangle \rho_{j, s}^{0} .
$$

В терминах поля

$$
\bar{A}^{\prime}=\bar{A}-\sum_{j=2}^{k} \frac{1}{\kappa^{j-1}} A^{j-1} \rho_{j}
$$

она принимает канонический вид

$$
\frac{1}{k} \omega=\int_{\Sigma_{g}}\left[\left\langle\delta A, \delta \bar{A}^{\prime}\right\rangle+2 \pi i \sum_{a=1}^{n} \delta\left\langle p_{a}, g_{a}^{-1} \delta g_{a}\right\rangle\right]
$$


Это свободная система с решениями

$$
A=A_{0}=\text { const }, \quad \bar{A}(\mathbf{t})=\bar{A}_{0}+\sum_{j=2}^{k} \frac{\left(A^{j-1}+\cdots\right)}{\kappa^{j-1}} \sum_{s=1}^{l_{j}} t_{j, s} \rho_{j, s}^{0} .
$$

После гамильтоновой редукции относительно калибровочной симметрии (4.40) мы получаем расширенное фазовое пространство

$$
\mathcal{P}_{k}^{N}=\left\{L, \bar{L}, \mathbf{p}, \rho_{j}, \quad j=2, \ldots, k\right\} .
$$

На критическом уровне получаем системы Хитчина с интегралами порядков $j=$ $2, \ldots, k$. При $k<N$ число интегралов меньше размерности конфигурационного пространства. При $k \geqslant N$ системы являются полностью интегрируемыми, хотя интегралы порядков $j>N$ не являются независимыми. Таким образом, выделенный случай соответствует $k=N$.

Интересно рассмотреть предел $k \rightarrow \infty$. Поскольку дифференциальные операторы произвольного порядка порождают алгебру Ли, $\mathcal{W}$-структура приобретает теоретико-групповую интерпретацию, как и в случае $\mathcal{W}_{2}$. Как мы указывали выше, одновременный предел $k \rightarrow \infty, N \rightarrow \infty$ является выделенным и может привести к интересным теориям поля.

Благодарности. Работа А. Л. частично поддержана грантами РФФИ № 98-0100344 и № 96-15-96455 поддержки научных школ. Работа М. О. частично поддержана грантами РФФИ № 96-02-18046, INTAS № 96-518 и № 96-15-96455 поддержки научных школ. Мы благодарны Институту Макса Планка в Бонне, где выполнялась эта работа, за гостеприимство.

\section{Список литературы}

[1] C. M. Hull. Lectures on $\mathcal{W}$-gravity, $\mathcal{W}$-geometry and $\mathcal{W}$-strings. hep-th/9302110.

[2] M.A. Gabeskeria, M.V. Saveliev. Lax type representation for the embeddings of Riemann manifolds. Preprint IHEP. Protvino: IHEP, 1983.

[3] M. Ugaglia. On the Hamiltonian and Lagrangian structures of time-dependent reductions of evolutionary PDEs. solv-int/9902006.

[4] N. Hitchin. Duke Math. J. 1987. V. 54. P. 91-114.

[5] B. van Geemen, E. Previato. On the Hitchin System. alg-geom/9410015.

[6] K. Gawedzki, P. Tran-Ngog-Bich. Hitchin systems at low genera. hep-th/9803101.

[7] N. Nekrasov. Commun. Math. Phys. 1996. V. 180. P. 587-604; hep-th/9503157.

[8] A. Levin, M. Olshanetsky. Am. Math. Soc. Transl. 2. 1999. V. 191.

[9] A. Beilinson, V. Drinfeld. Opers. Preprint, 1994.

[10] V. Fock. Towards the geometrical sense of operator expansions for chiral currents and $W$-algebras. Preprint ITEP. Moscow: ITEP, 1990; A. Bilal, V. Fock, Ia. Kogan. Nucl. Phys. B. 1991. V. 359. P. $635-672$.

[11] A. Gerasimov, A. Levin, A. Marshakov. Nucl. Phys. B. 1991. V. 360. P. 537-558.

[12] I. Krichever. Commun. Pure Appl. Math. 1994. V. 47. № 4. P. 437-475.

[13] В. И. Арнольд. Математические методы классической механики. М.: Наука, 1974. 